\title{
EFFECTS OF MEPROBAMATE, CHLORDIAZEPOXIDE, DIAZEPAM, AND SODIUM PENTOBARBITAL ON VISUALLY EVOKED RESPONSES IN THE TECTO- TEGMENTAL AREA OF THE RAT
}

\author{
M. E. Olds and G. BALDRIGHI \\ The University of Michigan, Ann Arbor, Michigan
}

(Accepted 27 August 1967)

\begin{abstract}
Summary-The effects of meprobamate, chlordiazepoxide, diazepam and sodium pentobarbital on sensory input to the boundary area between the dorsal midbrain tegmentum and ventral tectum of the rat have been investigated in order to find out whether effects obtained with these compounds in the hypothalamus can also be observed at the mesencephalic level. The following results have been obtained.
\end{abstract}

(1) At a dose of $2.5 \mathrm{mg} / \mathrm{kg}$ i.p., chlordiazepoxide had no effect; at 5, 10, 15, and 20 $\mathrm{mg} / \mathrm{kg}$, this compound decreased the amplitude of the initial components of the visually evoked responses in the rat. There was an increased effect at higher doses.

(2) Diazepam had effects similar to those produced by administration of chlordiazepoxide. The evoked responses were of smaller amplitude after injection of 5, 10, and 20 $\mathrm{mg} \mathrm{kg}$ i.p. At the highest dose, diazepam caused larger reductions in amplitude than chlordiazepoxide at $20 \mathrm{mg} / \mathrm{kg}$.

(3) Meprobamate at 40,80 and $100 \mathrm{mg} / \mathrm{kg}$ i.p. increased the amplitude of the evoked responses. There was more individual variation with this compound than with chlordiazepoxide, and more waxing and waning of the effects, but little difference in the magnitude of facilitation at the various doses. At a dose of $120 \mathrm{mg} / \mathrm{kg}$, the size of the evoked responses decreased.

(4) At doses of 5, 10, and $20 \mathrm{mg} \mathrm{kg}$ i.p., sodium pentobarbital caused increases in the amplitude of the evoked responses. Only at a dose of $20 \mathrm{mg} / \mathrm{kg}$ was there a slight decrease in amplitude, lasting only $25 \mathrm{~min}$ after drug administration. Thereafter, responses to this dose were larger than control responses, but not as large as responses to the two smaller doses.

The significance of these results is discussed in the context of earlier data on the effects of these compounds and the barbiturates on sensory input to the midbrain reticular formation. A difference in mode of action, at low doses, is shown by the data. A relationship between the effects of meprobamate and the barbiturates on sensory input in the rat finds support in these results.

\section{INTRODUCTION}

THE PHARMACOLOGICAL effects of meprobamate and chlordiazepoxide on the midbrain reticular formation (MRF) have been investigated to determine whether the sedative properties of these compounds are due to depression of MRF activity. This question has not yet been unequivocally answered. Some investigators have claimed that meprobamate (MPB) has no significant effect.either on EEG arousal induced by RF stimulation or on EEG frequency (SCHALLEK and KUEHN, 1965), and that doses of $40-80 \mathrm{mg} / \mathrm{kg}$, rather than depressing evoked potentials in the MRF, increase their amplitude: this suggests increased rather than depressed RF activity (DOMINO, 1962; KILlam, 1962; KLETZKIN and SwAN, 
1959). Sodium pentobarbital, on the other hand, and barbiturates in general, induce EEG synchrony and raise thresholds for EEG arousal caused by MRF stimulation (Domino, 1962; KILlam, 1962). On the basis of this and other available data, MPB is thought to cause sedation via action on the thalamic, limbic and hypothalamic structures at doses that leave MRF activity relatively unaffected. Depressant action by MPB on locomotor activity and on spinal reflexes induced by MRF stimulation is generally acknowledged, but evidence about its effect at relatively low doses on the cephalic outflow of the MRF remains inconclusive. Others have claimed that MPB is perhaps less potent than some of the barbiturates in depressing MRF activity, but the data (CHIN et al., 1965; DomINO, 1962) suggest that the actions of MPB and barbiturates are similar rather than different.

Chlordiazepoxide (CDP) is differentiated from the barbiturates on somewhat similar grounds, although there is no conclusive evidence on whether MPB and CDP are like each other but different from the barbiturates, alike in action and similar to the barbiturates, or different from one another and from the barbiturates.

Randall (1959); SChallek and Kuehn (1965); Morillo (1962) and others (Domino, 1962; KILLAM, 1962) have reported that CDP and diazepam, a drug closely related to CDP, have no significant effect on the thresholds at which electrical stimulation in the MRF produces EEG arousal or behavioral arousal. SCHALLEK and KUEHN (1965), however, noted an increase in EEG frequency and behavioral restlessness; WHITE et al. (1965) reported EEG synchrony, accompanied, at low doses, by an increase in evoked activity in the MRF. In rats in which escape behavior was induced by electrical stimulation of the MRF, CDP at $5 \mathrm{mg} / \mathrm{kg}$ had less depressive effect than MPB (OLDs, 1966). Both drugs have some sedative action, but it is not clear whether this action is like that of the barbiturates, White et al. (1965) or essentially different, whether it depresses the MRF or leaves it relatively unaffected.

The present study was carried out in hope of finding a definite answer. The electrodes were aimed at the most ventral layer of the tectum. In a majority of cases, the visually evoked responses were recorded from a boundary area between the ventral tectum and the dorsal midbrain tegmentum, lateral to the central gray (Fig. 1). Two reasons determined the choice of this particular locus for recording of sensory inflow. One, that it would be useful to search for differences between the effects of MPB and CDP on the initial components of the visual evoked responses in the MRF with the use of averaging techniques. The recording electrodes, though at the dorsal boundary of the MRF, were nevertheless still within the activating system. Earlier work on rats with chronically implanted electrodes in this tecto-tegmental area showed that electrical stimulation at these points caused cortical low amplitude, high frequency waves to replace EEG patterns normally associated with sleep (OLDS and PERETZ, 1960). Therefore, it seemed that effects on sensory inflow to this tecto-tegmental area with MPB and CDP could be related to previous work with these drugs on the MRF. An additional reason for the choice of the tecto-tegmental area as the recording site was that electrical stimulation of points in this region causes behavioral escape (OLDS and OLDS, 1963); MPB and diazepam inhibited escape at lower doses than CDP (OLDs, 1966). Recording sensory input to this region thus provided the opportunity to obtain data both on the mode of action of these compounds on the reticular activating system and on a motivational system. A comparison of data obtained by means of summating technique in unanesthetized rats raised the possibility of finding differences between these compounds significant enough to give a clearer understanding of their action in the CNS. 


\section{METHODS}

Five electrodes, $250 \mu$ in diameter, were implanted in the central nervous system of each of 16 male albino rats weighing 300-350 g each. Surgery was performed under sodium pentobarbital anesthesia $(30 \mathrm{mg} / \mathrm{kg}$ i.p.). The method of implantation has previously been described (OLDS, 1966). In all subjects, all 5 electrodes were aimed at the tecto-tegmental area, ${ }^{*}$ in close proximity to the central gray. The most medial probe was $1 \mathrm{~mm}$ lateral to the midline, and each subsequent one was placed $\frac{1}{2} \mathrm{~mm}$ more laterally, but at the same depth and along the same dimension on the longitudinal axis. The fifth electrode, its entire shaft uninsulated, was placed $1 \frac{1}{2} \mathrm{~mm}$ laterally from the fourth probe. The various probes formed a multi-pronged array of identical length and distance from one another, except that the one placed most laterally was always selected as the indifferent electrode for monopolar recordings. Histological verification showed that all electrodes aimed at the tecto-tegmental area were correctly placed, with some very slight variation in depth (Fig. 1).

Some of the subjects had been used in studies of the alteration of visually evoked responses (VER) over time and were accustomed to the experimental situation. Before the present investigations, none had received drugs, except during surgery. Each animal over a period of 4 months, had been the subject of at least six extensive periods of recording of VER.

Recordings of VER began 1 week after surgery. Four rats were studied at a time, one placed in each of the arms of a cross-shaped, semi-soundproof, temperature-controlled chamber. Each subject was gently restrained in a small box.

The walls of the chamber were lined with mirrors to minimize the effects of changes in orientation. In the center of the chamber were a stroboscope unit and several small speakers delivering white masking noise. The head of each subject was approximately 8 in. from the light source.

The visual stimuli were single flashes of white light from a Grass photic stimulator. Each was $10 \mu \mathrm{sec}$ long and had a relative intensity of 375,000 candle power. Stimuli were automatically programmed to be presented at an average interval of $48 \mathrm{sec}$ continuously throughout the test, even when recording was interrupted for $1 \mathrm{~min}$ so that data for the preceding block of 20 stimuli could be graphed.

Recordings were monopolar; the tip of the recording probe was $21 \mathrm{~mm}$ from that of the indifferent one. Since each subject had four equi-distant probes, the electrode yielding the largest response was selected as the recording probe. In the dorsal midbrain tegmentum, the largest responses were obtained from the most medial electrode.

Evoked activity before and after each photic stimulus was recorded on a Grass EEG, four channels of which fed into a Mnemotron 400-A Computer of Average Transients (CAT). The responses evoked from one subject were recorded on each channel. Time per analysis on the CAT was $500 \mathrm{msec}$. Visual stimuli were presented $100 \mathrm{msec}$ after analysis began. Each set of 20 visual stimuli constituted one series; the responses to this series were summed by the CAT, and the resulting analogue readout was plotted as a curve by an X-Y plotter (Fig. 1). The evoked responses to each block of 20 trials were analysed in terms of the amplitude of the first biphasic wave of each response (Fig. 1).

*The terms midbrain tegmentum and tectum or tecto-tegmental region are used to refer to approximately the same structure in the rat. They refer to the anatomical area lateral to the central gray and caudal to the posterior commissure. Stimulation of this structure, even at low levels, produces increased attention and behavioral arousal. 
The test lasted about $11 \mathrm{hr}$; for the first $4 \mathrm{hr}$ the subjects were presented the stimuli but no drug was administered. Then a saline or glycol solution injection was administered, and again at least six read-outs were taken to determine the effects of solvent injection. This was then followed by drug administration.

A mean control amplitude was computed from the amplitudes of the averaged responses during the last four blocks of 20 trials. Differences between mean control amplitudes of responses and amplitude values were obtained for $25,50,75,100,125,150,175$, and 200 min after drug administration. Differences in amplitudes of VER after medication were expressed as percentages of control values.

The drugs used were chlordiazepoxide hydrochloride at doses of $2 \cdot 5,5,7 \cdot 5,10,15$, and $20 \mathrm{mg} / \mathrm{kg}$, meprobamate at doses of $40,80,100,120 \mathrm{mg} / \mathrm{kg}$, diazepam at doses of 5,10 , $20 \mathrm{mg} / \mathrm{kg}$, and sodium pentobarbital at doses of $5,10,20 \mathrm{mg} / \mathrm{kg}$. All drugs were administered intraperitoneally either in a physiological saline solution or in a polyethylene glycol 400 suspension. The concentration of each drug was such that the subjects received an equal volume per kilogram of body weight. If subjects were to be used more than once, then at least 1 week intervened between administrations of a drug. Statistical analyses (SIEGEL, 1956) were performed to determine the effects of different drugs, or doses of the same drug, on each group of animals (U. Mann-Whitney Test).

\section{RESULTS}

At all effective doses, CDP and diazepam decreased the amplitude of the evoked responses, whereas MPB and sodium pentobarbital increased the amplitudes, except at very high doses.

As Table 1 shows, CDP at a dose of $2.5 \mathrm{mg} / \mathrm{kg}$ caused almost no change in the size of the responses during a 200 -min test period. The mean percentage value of the average responses for a group of 4 rats is 100 . At a dose of $5 \mathrm{mg} / \mathrm{kg}$, the VER decreased by $10 \%$ during the first $25 \mathrm{~min}$. The effect waxed and waned; however, an effect of the same magnitude as that shown initially, a $10 \%$ decrease from control values, was also shown 175 min after drug administration. As the doses increased, the effect gradually increased, especially during the first $25 \mathrm{~min}$, so that after $20 \mathrm{mg} / \mathrm{kg}$ there was a reduction of $20 \%$ in the VER.

Doses of $7.5 \mathrm{mg} / \mathrm{kg}$ and $10 \mathrm{mg} / \mathrm{kg}$ produced about the same effects. As the dose became larger, the effect not only became more pronounced but lasted longer; some waxing and waning of the magnitude occurred at every dose. Surprisingly, the magnitude of the effect $200 \mathrm{~min}$ after drug administration is either identical (at $20 \mathrm{mg} / \mathrm{kg}$ ) or very close in value to that shown during the first $25 \mathrm{~min}$, whereas 75 to $100 \mathrm{~min}$ after injection there was a reduction of drug effects. The mean percentages of change for all groups of rats, at different doses, during the $200 \mathrm{~min}$ after drug administration are plotted against dose in Fig. 2. The magnitude of the effect is almost identical at $5,7 \cdot 5$, and $10 \mathrm{mg} / \mathrm{kg}$; at high doses the effects increase markedly. It may be noted that all effects are decrements of the VER. For example, at a dose of $20 \mathrm{mk} / \mathrm{kg}$, seven out of eight responses had decreased; after $175 \mathrm{~min}$ all eight responses were again smaller than the control responses. Even at a dose of $5 \mathrm{mg} / \mathrm{kg}$ of CDP, seven responses were smaller during the first $25 \mathrm{~min}$, six were smaller during the interval between 50 and $75 \mathrm{~min}$, and all eight responses were smaller during the interval between 50 and $75 \mathrm{~min}$; and all eight responses were smaller during the interval between 150 and $175 \mathrm{~min}$. Although in some individual cases the VER at a 


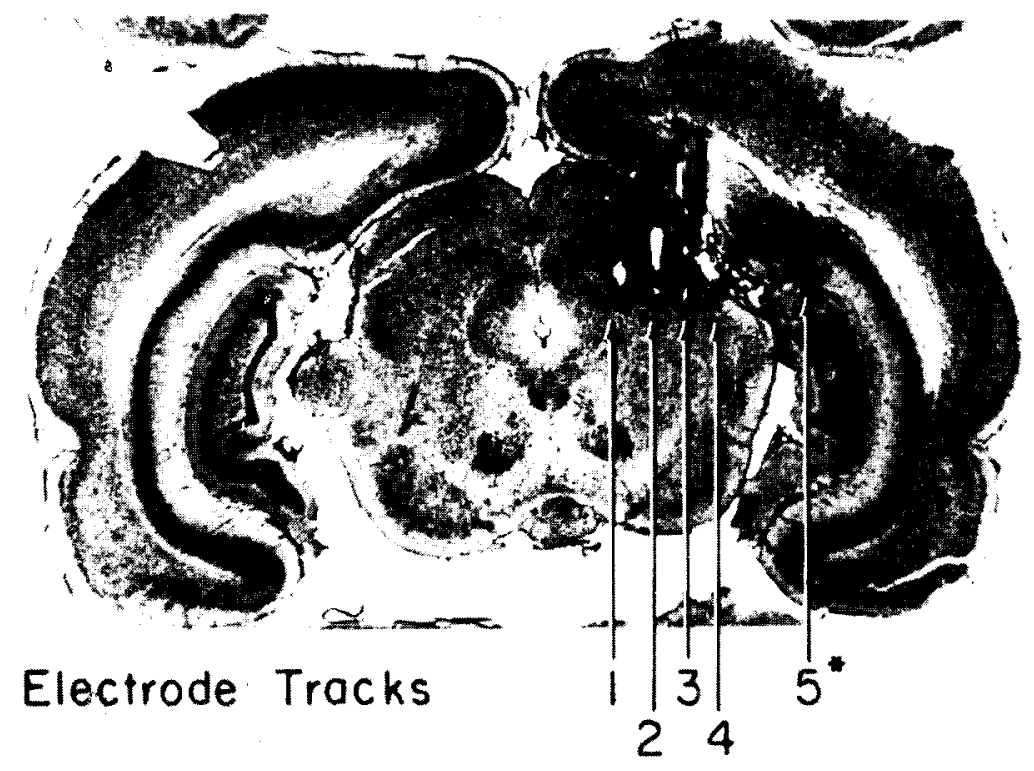

Fig. I. Rat 3036 dorsal midbrain tegmentum. Electrode No. 5 uninsulated along its entire shaft, used as indifferent probe. 